\title{
MicroRNA-30a-3p inhibits the progression of lung cancer via the PI3K/AKT by targeting DNA methyltransferase 3a
}

This article was published in the following Dove Press journal: OncoTargets and Therapy

\author{
Desheng Wei ${ }^{1}$ \\ Guangmao $\mathrm{Yu}^{\prime}$ \\ Yeping Zhao ${ }^{2}$
}

'Department of Thoracic Surgery, Shaoxing People's Hospital, Shaoxing Hospital of Zhejiang University, Shaoxing, Zhejiang 3I2000, People's Republic of China; ${ }^{2}$ Department of B-Ultrasonic Room, Shaoxing People's Hospital, Shaoxing Hospital of Zhejiang University, Shaoxing, Zhejiang 3/2000, People's Republic of China
Correspondence: Yeping Zhao Department of B-ultrasonic Room, Shaoxing People's Hospital, Shaoxing Hospital of Zhejiang University, No.568 Zhongxinbei Road, Shaoxing, Zhejiang 312000, People's Republic of China

Tel +86 I 3675756377

Email yeping2_sxph@I63.com
Background: MicroRNAs (miRNAs) are small non-coding RNAs, involved in pathological and physiological processes via regulating target genes expression. Abnormally expressed miR-30a-3p has been verified in several tumors, such as liver cancer, esophageal cancer and lung cancer. It was reported that DNA methylation plays a critical role in the tumorigenesis of lung cancer through regulated tumor suppressor genes silencing. Nevertheless, the potential mechanism of miR-30a-3p in restoring abnormal DNA methylation patterns is still unclear in lung cancer. Therefore, because the miR-30a-3p is complementary to the 3'untranslated regions (3'-UTR) of DNA methyltransferase 3A (DNMT3A), we investigated whether miRNA-30a-3p could target DNMT3a to regulate the progression of lung cancer cell.

Methods: qRT-PCR was used to evaluate miR-30a-3p and DNMT3a mRNA expression levels in A549 lung cancer cells and normal cell line BEAS-2B. MiR-30a-3p expression plasmid was transferred into A549 cells. The target of miR-30a-3p was detected by luciferase reporter assay. Western blot was used to measure related protein expression levels. MTT assay was used to measure the proliferation of cells in each group. The cycle and apoptosis of cells were detected by flow cytometry.

Results: We found down-regulation of miR-30a-3p mRNA expression and up-regulation of DNMT3a mRNA expression in A549 cells. Overexpression of miR-30a-3p downregulates DNMT3a or blocked DNMT3a by interference vector, significantly inhibited the proliferation and G1/S transition in A549 cells via regulating p38 MAPK pathway, and induced the apoptosis in A549 cells via regulating Bcl-2/Bax protein levels. Furthermore, we observed the opposite phenomenon in A549 cells transfected with both miR-30a-3p and DNMT3a vector.

Conclusion: Our data show that miR-30a-3p suppressed the progression of lung cancer via regulating p38 MAPK pathway by targeting DNMT3A in A549 cells, indicating that miR$30 a-3 p$ might be a novel potential therapeutic strategy in the treatment of lung cancer.

Keywords: DNA methylation, MiR-30a-3p, lung cancer, DNA methyltransferases, tumor suppressor genes

\section{Introduction}

DNA methylation as a conserved epigenetic silencing mechanism in mammalian cells is involved in numerous biological processes. ${ }^{1}$ Abnormal DNA methylation regulates the expression of tumor suppressor genes (TSGs) or oncogenes, of which translating proteins participate in genomic instability, malignant cell growth, metastasis and differentiation. ${ }^{2,3}$ It has been well known that aberrant DNA 
hypermethylation in TSGs leads to inhibits of transcription and induce in tumorigenesis. DNA methylation is carried out by DNA methyltransferases (DNMT). Among all members of DNMT family, abnormal DNMT3a expression has been reported in many types of tumors. ${ }^{4,5} \mathrm{~A}$ number of reports have confirmed the overexpression of DNMT3a mRNA or protein levels in human tumors, including hepatocellular carcinomas, ${ }^{6}$ prostate cancer, ${ }^{7}$ non-small cell lung carcinoma ${ }^{8}$ and breast cancer. ${ }^{9}$ However, the potential mechanism of DNMT3a in lung cancer needs further study.

MicroRNAs (miRNAs) are a class of 12-25-nucleotide endogenous non-coding RNAs that cause mRNA degradation or inhibiting translation by interacting with the $3^{\prime}$ untranslated region ( $3^{\prime}$-UTR) of the target genes. ${ }^{10}$ MiRNA play important regulatory role in various fundamental biological processes, such as development, differentiation and apoptosis. ${ }^{11,12}$ Furthermore, miRNAs can act as either oncogenes or tumor suppressors, and due to its potential on regulation of numerous genes, miRNAs represent powerful discovery tools to pioneer new ways impacting cancer. ${ }^{13}$ MiR-30a as a tumor suppressor can regulate proliferation, apoptosis, invasion and migration of various tumor cells. ${ }^{14,15}$ MiR-30a can attenuate the progression of breast cancer by inhibiting the expression of the downstream target gene Notch1. ${ }^{16}$ In H. pylori gastric cancer models, miR-30a inhibited tumor growth via targeting COX-2 and BCL-9. ${ }^{17}$ MiR-30a-3p as a member of miR30a family has been reported to be down-regulated in several tumors. ${ }^{18}$ Qi et al reported that down-regulation of miR-30a-3p/5p promotes esophageal squamous cell carcinoma (ESCC) cell proliferation by activating the Wnt signaling pathway. ${ }^{19}$ However, little is known about the role and underlying molecular mechanism of action of miR-30a-3p in lung cancer.

In this study, we investigate the effect and the potential mechanism of miR-30a-3p in the progression of lung cancer. The results indicated that miR30a-3p suppresses the development of A549 cells by targeting DNMT3a via the p38 MAPK pathway. These findings suggest miR-30a$3 p$ have value as therapeutic strategies for lung cancer and warrants continued investigation in this regard.

\section{Materials and methods}

\section{Cell culture}

A549 (BNCC337696) and BEAS-2B (BNCC100240) cells were obtained from BeNa Culture Collection. Cells were cultured in RPMI-1640 medium (ThermoFisher, Waltham, USA) supplemented with $10 \%$ fetal bovine serum (FBS, Gibco, ThermoFisher, Waltham, USA) under the condition with $95 \%$ air and $5 \% \mathrm{CO}_{2}$ at $37^{\circ} \mathrm{C}$ in a humidified chamber.

\section{Plasmid constructions and transfection}

The pc-DNA ${ }^{\mathrm{TM}} 6.2-\mathrm{GW} / \mathrm{EmGFP}-\mathrm{miR}$ vector (Invitrogen, Waltham, USA) was used to generate vectors of re-expression of miR-30a-3p and DNMT3a. The gene coding for miR-30a-3p was chemically synthesized and cloned in to pc-DNA ${ }^{\mathrm{TM}} 6.2-\mathrm{GW} /$ EmGFP-miR vector between the EcoRI and HindIII sites using sense primer 5'-TGCC CTGG CTTAGTTATCACAGTGCTGATGCTGTCTACT CTAAAGCTAGAGTACTGTGAT AACTCAAGGATGG CA-3' and anti-sense primer 5'-TGCCATCCTTGAGTTA GTT ATCACACTACTGTACCTTTAGATTAGTCAGCA TCAGCACAGTGATAACTGAGCCAGGGCA-3'. Target Plus Smart pool siRNAs (Dharmacon, Waltham, USA) containing a mixture of oligonucleotides with potential for DNMT3a abrogation was used to silence DNMT3a expression. The luciferase-UTR reporter constructions which were generated by introducing the wild-type (wt)/ mutant (mut) EGFR 3'-UTR carrying a putative miR-30a$3 p$ binding site were used to identify the DNMT3a by miR-30a-3p targeting.

\section{Reverse transcription-quantitative polymerase chain reaction (RT-qPCR)}

Total RNA was extracted by TRIzol reagent (Takara, Dalian, China) according to the manufacturer's instructions, and RNAse-free DNase was used to remove DNA contamination. RNA was quantified by an ultraviolet spectrophotometer and $1 \%$ agarose electrophoresis. $1 \mu \mathrm{g}$ RNA from each sample was reverse transcribed to obtain cDNA by using PrimeScript ${ }^{\mathrm{TM}}$ RT reagent kit (Tkara). The expression of relative genes was analyzed using $2 \times$ SYBR Premix ExTaqTM (Takara) and the specific primers were miR-30a-3p forward: 5'-CCCTGCTCTGGCTGGTCAAA CG GA-3', miR-30a-3p reverse: 5'-TTGCCAGCCCT GCTGTAGCTGGTTGAAG-3' and DNMT3a forward: 5'CGGCAGAATAGCCAAGTTCA-3', miR-30a-3p reverse: 5'-GGGAAGCCAAACACCCTTTC-3'. The threshold cycle $(\mathrm{Ct})$ was determined using $\Delta \Delta \mathrm{Ct}$ method, which makes $\mathrm{Ct}$ values normalized to the endogenous control gene $(G A P D H)$. For each group, the quantification was triplicated. 


\section{Dual luciferase assay}

A549 cells were co-transfected with 500 ng DNMT3a-3'UTR (wild type and mutant type) and $50 \mathrm{nM}$ miR-30a-3p mimics or $100 \mathrm{nM}$ inhibitor by using Lipofectamine 2000 (Invitrogen, USA) according to the manufacturer's instructions. After $48 \mathrm{hrs}$, cells were lysed to DualLuciferase Reporter Assay System (Promega, Madison, WI, USA), and luciferase activity was detected by GloMax20/20 Luminometer (Promega). Luciferase activity was normalized by Renilla/Firefly luciferase signal in lymphocytes.

\section{Western blot}

For total protein extraction, cells were washed with phosphate-buffered saline (PBS) and lysed with RIPA buffer (Beyotime, Shanghai, China) supplemented with protease inhibitor. Cell lysate was centrifuged at $12,000 \times \mathrm{g}$ for 15 mins and the supernatants were collected. The concentration of proteins was measured by BCA kit. $30 \mu \mathrm{g}$ protein in each group were separated by $10 \%$ SDS-polyacrylamide gels. After electrophoresis at $200 \mathrm{~mA}$ for 2 hrs, proteins were transferred onto PVDF membrane (Millipore, USA). The membranes were blocked with $5 \%$ skim milk for $2 \mathrm{hrs}$ at room temperature. Subsequently, the membrane was incubated with primary antibodies against DNMT3a (ab188470, 1:2000 dilution), p-38 (ab27986, 1:1000 dilution), p-p38 (ab178867, 1:1000 dilution), p21CIP1 (ab212247, 1:1000 dilution), p27KIP1 (ab32034, 1:5000 dilution), cyclin-dependent kinase 2 (CDK2) (ab32147, 1:2000), cyclin D (ab134175, 1:10,000), Bcl-2 (ab32124, 1:1000) and Bax (ab32503, 1:2000 dilution) overnight at $4^{\circ} \mathrm{C}$. AntiGAPDH (ab128915, 1:10,000) antibody was selected as internal reference. Then, the membranes were washed with Tris-buffered saline and incubated in biotinylated goat IgG secondary antibody (ab7090) for $2 \mathrm{hrs}$ at room temperature. All antibodies were purchased from Abcam (USA). Immunoreactivity was visualized by colorimetric reaction using ECL substrate buffer (Millipore, Massachusetts, USA). Membranes were scanned with Gel Doz EZ imager (Bio-rad, USA).

\section{MTT assay}

The proliferation of A549 cells was measured by MTT colorimetric method. Cells were seeded in 96-well cell culture plates with $1 \times 10^{4}$ cells per well. After treatment for $24 \mathrm{hrs}, 48 \mathrm{hrs}$ and $72 \mathrm{hrs}$, cells were incubated with
$20 \mu \mathrm{L}$ MTT for $2-4 \mathrm{hrs}$. Then, the purple precipitate was visible, the medium was removed and $150 \mu \mathrm{L}$ DMSO was added into each well. After shaking at low speed for 10 mins, the absorbance was recorded at 570 $\mathrm{nm}$. For each detect, the total procedure was repeated 3 times.

\section{Cell cycle analysis}

After treatment for $48 \mathrm{hrs}$, cells were collected by trypsinization, and $1 \times 10^{6}$ cells were used for analysis. The cells were washed twice with PBS and fixed in ice-cold ethanol overnight at $4{ }^{\circ} \mathrm{C}$. Then, the cells were washed with PBS and incubated in $1 \mathrm{~mL}$ staining solution (10 U/mL RNaseA and $20 \mu \mathrm{g} / \mathrm{mL}$ propidium iodide) for 30 mins at room temperature. Cell cycle distributions were assayed by fluorescence-activated cell sorting using a flow cytometer (FACSort; BD Biosciences, Franklin Lakes, USA).

\section{Cell apoptosis assay}

The apoptosis of A549 cells was detected by AnnexinVFITC/PI (propidium iodide) flow cytometry kit (BD) according to the manufacturer's instructions. Cells were washed twice with ice-cold PBS and resuspend $200 \mu \mathrm{l}$ of binding buffer at a concentration of $1 \times 10^{6}$ cells $/ \mathrm{mL}$. Cells were incubated in $10 \mu \mathrm{L}$ PI and $10 \mu \mathrm{L}$ Annexin V-FITC for 30 mins at $4^{\circ} \mathrm{C}$ in the dark. Finally, $300 \mu \mathrm{l}$ binding buffer was added and analyzed by flow cytometry (Beckman Coulter, Cytomics FC 500, CA) within $1 \mathrm{hr}$.

\section{Statistical analysis}

All numerical data were expressed as mean \pm S.D. The statistical differences were analyzed by one-way analysis of variance (ANOVA) using SPSS 19.0 software package. Differences were considered as statistically significant at $p<0.05$ and very significant at $p<0.01$.

\section{Results}

\section{The expression of miR-30a-3p deceased, while DNMT3a increased in the A549 cells}

We detected the miR-30a-3p and DNMT3a expressions in A549 cells and normal BEAS-2B cells. As shown in Figure 1A, the level of miR-30a-3p in A549 cells was lower than that in BEAS-2B cells. The DNMT3a mRNA (Figure 1B) and protein (Figure 1C and D) levels in A549 

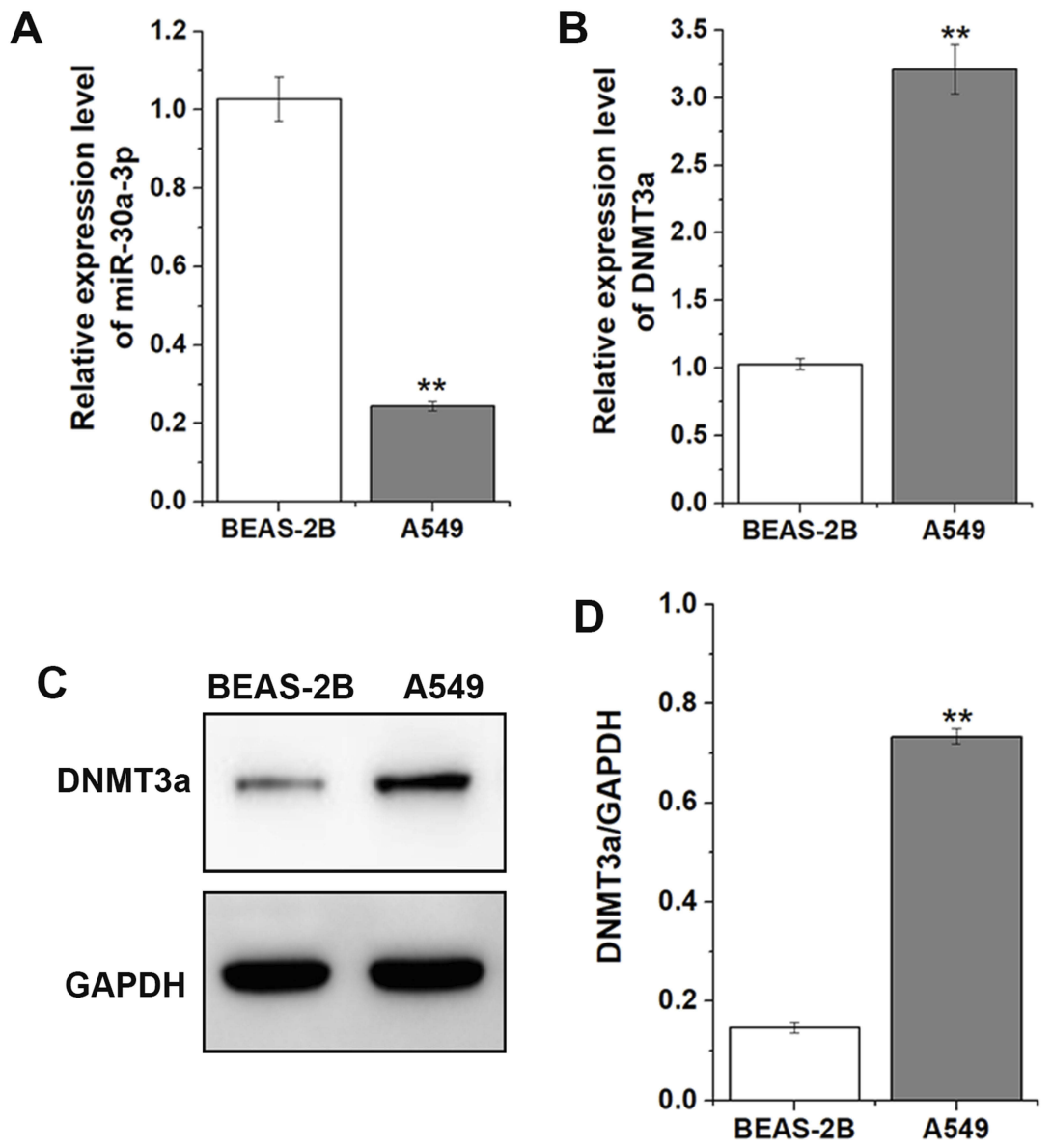

Figure I The differential expression of miR-30a-3p and DNMT3a in lung cancer A549 cells and normal BEAS-2B cells. (A) The mRNA levels of miR-30a-3p in A549 cells and normal BEAS-2B cells. (B) The mRNA levels of DNMT3a in A549 cells and normal BEAS-2B cells. (C, D) Western blot analysis of DNMT3a protein expression in A549 cells and normal $B E A S-2 B$ cells. Data are reported as mean $\pm S D(n=3)$. $* * P<0$.I versus $B E A S-2 B$ cell group.

cells increased significantly compared with that in BEAS-2B cells. These data suggest that miR-30a-3p and DNMT3a might involve the progression of A549.

\section{miR-30a-3p targets DNMT3a}

We constructed the miR-30a-3p overexpression vector and transfected with A549 cells. The results showed that the expression of miR-30a-3p increased significantly in A549 cells after transfection (Figure 2A). The protein expression levels of DNMT3a in A549 cells transfected with miR30a-3p overexpression vector decreased significantly (Figure 2B and C). Moreover, we co-transfected A549 cells with miR-control, miR-30a-3p mimics, miR-30a-3p inhibitor and pmirGLO-DNMT3a-3'-UTRwt or pmirGLO-DNMT3a-3'-UTR-mut. We found that miR-30a-3p inhibited the firefly luciferase activity of pmirGLO-DNMT3a-3'-UTR-wt, whereas miR-control did not (Figure 2D).
miR-30a-3p inhibits the growth of A549 cells by suppressing the $\mathrm{p}-38$ MAPK signaling pathway

To study the effect of miR-30a-3p on A549 cell proliferation, we performed MTT and cell cycle assays. We found that upregulation of miR-30a-3p significantly inhibited the proliferation of A549 cells at $48 \mathrm{hrs}$ and $72 \mathrm{hrs}$ (Figure 3A). Up-regulation of miR-30a-3p resulted in a significant repression from the $\mathrm{G} 1$ phase to $\mathrm{S}$ phase and $\mathrm{G} 2$ phase in A549 cells (Figure 3B). Furthermore, we analyzed the expression of cell cycle regulators related with the p-38 MAPK pathway. The results demonstrated that the p-38 MAPK pathway was activated by transfection with miR-30a-3p (Figure 3C), which induced the expression of p21CIP1, p27KIP1 and inhibited the expression of CDK2, cyclin D in A594 cells (Figure 3D). Those results suggest that miR-30a-3p arrested the cell cycle at the G1 phase and suppressed cell proliferation in vitro. 

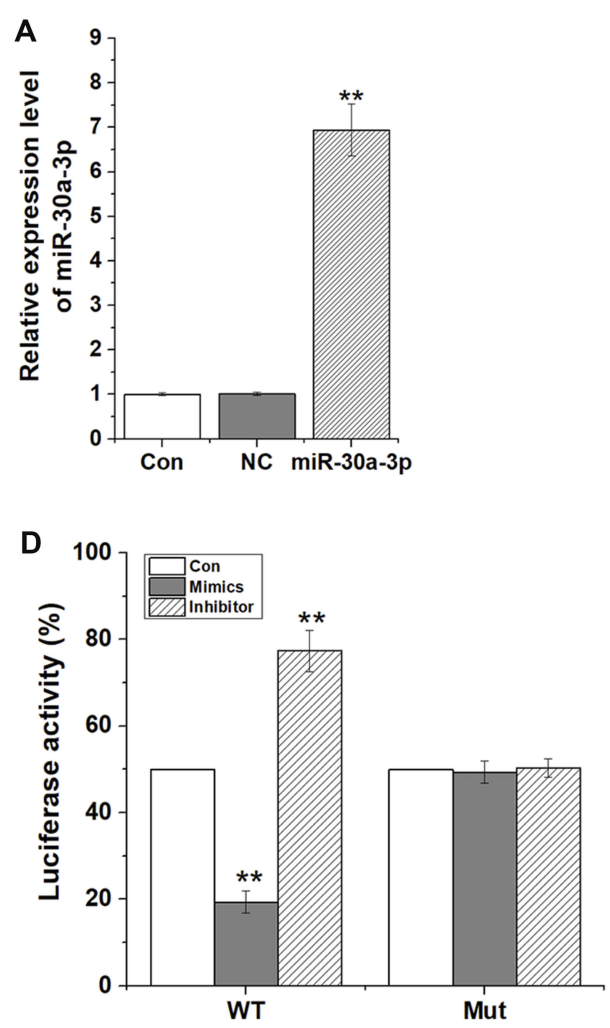

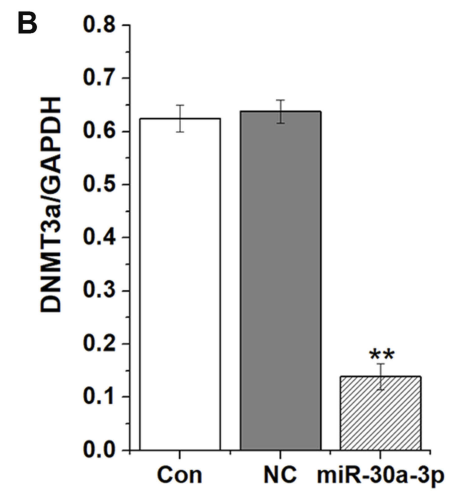

C

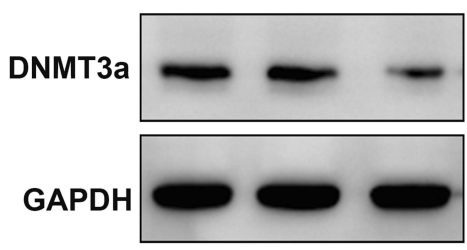

Figure 2 miR-30a-3p targets DNMT3a. (A) The mRNA levels of miR-30a-3p in A549 cells measured by RT-qPCR 48 hrs after transfection with miR-30a-3p. (B, C) Overexpression of miR-30a-3p inhibited the protein expression levels of DNMT3a in A549 cells. Data are reported as mean \pm SD $(n=3)$. $* *<<0.1$ versus $N C$ group. $(\mathbf{D})$ Luciferase assay miR-30a-3p binding sites in the WT-3'UTR and Mut-3'UTR of DNMT3A were cloned into pmirGLO Dual-Luciferase miRNA Target Expression vector. The normalized firefly luciferase activity was obtained by firefly luciferase activity/Renilla luciferase activity. Data are reported as mean \pm SD ( $n=3$ ). $* * P<0.1$ versus Con group. Abbreviations: Con, control group; NC, cells transfected with miR-control vector group; miR-30a-3p, cells transfected miR-30a-3p vector group.

\section{miR-30a-3p induces apoptosis in A549 cells}

The effect of miR-30a-3p on A549 cell apoptosis was detected by our in vitro experiments, the results indicating up-regulation of miR-30a-3p-induced cell apoptosis. Compared with cells in control group, miR-30q-3p overexpression-vector-transfected cells exhibited higher apoptosis rates (Figure 4A). In addition, we observed that miR-30a-3p regulated the expression of the proteins of apoptosis-related genes. As a result, up-regulation of miR-30a-3p promoted apoptosis by inactivating Bcl-2 and accelerating Bax (Figure 4B).

\section{DNMT3a silencing suppresses A549 cell development and up-regulating DNMT3a suppresses miR-30a-3p-induced A549 cell cycle arrest and apoptosis}

As reported previously, upregulation of miR-30a-3p affects cell proliferation, cycle and apoptosis in A549 cells. We identified that DNMT3A was a direct target of miR-30a-3p. Therefore, we silenced or activated the expression of DNMT3A via RNA interference or overexpression vector to confirm that DNMT3A is involved in the antitumor effects of miR-30a-3p. RNA interference or overexpression vector significantly silenced or activated the protein expression of DNMT3a (Figure 5A and B). Moreover, silencing DNMT3A significantly suppressed cell growth, arrest of the G1 phase and inhibited apoptosis (Figure $5 \mathrm{C}-\mathrm{G}$ ). These results follow the same trend as those obtained with miR-30a-3p-transfected A549 cells, but this effect was reduced by up-regulation of DNMT3a.

In addition, we examined the expression genes associated with cell cycle and apoptosis. As shown in Figure $5 \mathrm{H}$ and $\mathrm{I}$, the protein levels of the cell cycle regulators CDK2 and cyclin D were reduced by siDNMT3a, whereas those expressions were increased by up-regulation of DNMT3a. Moreover, siDNMT3a promoted cell apoptosis by inactivating the anti-apoptotic protein $\mathrm{Bcl}-2$ and activating the pro-apoptotic protein 

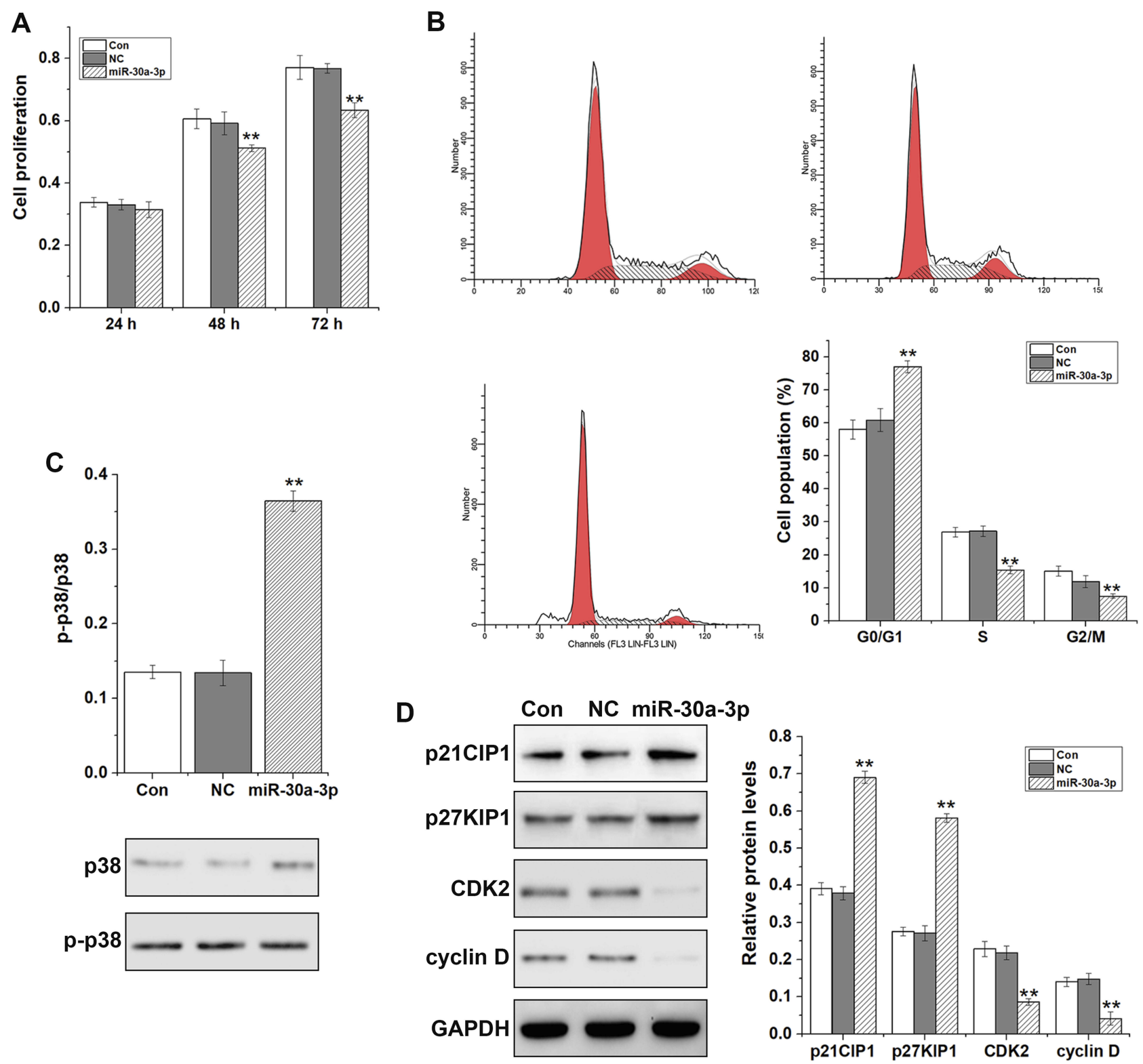

Figure 3 miR-30a-3p inhibited A549 cell proliferation and induced GI-phase arrest in vitro. (A) The effects of miR-30a-3p on A549 cell proliferation were evaluated by MTT assay at 24, 48 and $72 \mathrm{hrs}$. (B) The cell cycle was determined in A549 cells $48 \mathrm{hrs}$ after transfection. (C, D) Analysis of the expression of cell cycle regulatory protein in A549 cells following transfection with miR-30a-3p overexpression vector. Data are reported as mean $\pm S D(n=3)$. $* * p<0.1$ versus $N C$ group.

Abbreviations: Con, control group; NC, cells transfected with miR-control vector group; miR-30a-3p, cells transfected miR-30a-3p vector group.

Bax. Compared with miR-30a-3p transfected A549 cells, the cell cycle regulators CDK2 and cyclin D and antiapoptotic protein Bcl-2 increased significantly in A549 cells transfected with miR-30a-3p and DNMT3a, and the pro-apoptotic protein Bax decreased significantly.

\section{Discussion}

Downregulation of miR-30 family has been frequently reported in various tumors, including lung, liver, prostate and thyroid, in a large-scale miRNA expression arrays. ${ }^{20-22}$
MiR-30a-3p is a member of the miR-30 family, and has been demonstrated to be downregulated in several tumors, such as colorectal cancer, breast cancer and ovarian cancer. ${ }^{23-25}$ Consistent with the above results, we found that miR-30a-3p is downregulated in lung cancer A549 cells, and miR-30a-3p exert anti-lung cancer tumor effect by targeted DNMT3a to induced cell apoptosis and inhibited cell proliferation and cycle. Apart from the expression pattern, it has been observed that miR-30a-3p exhibited multiple roles in the regulation of tumor progression. MiR-30a-3p is 

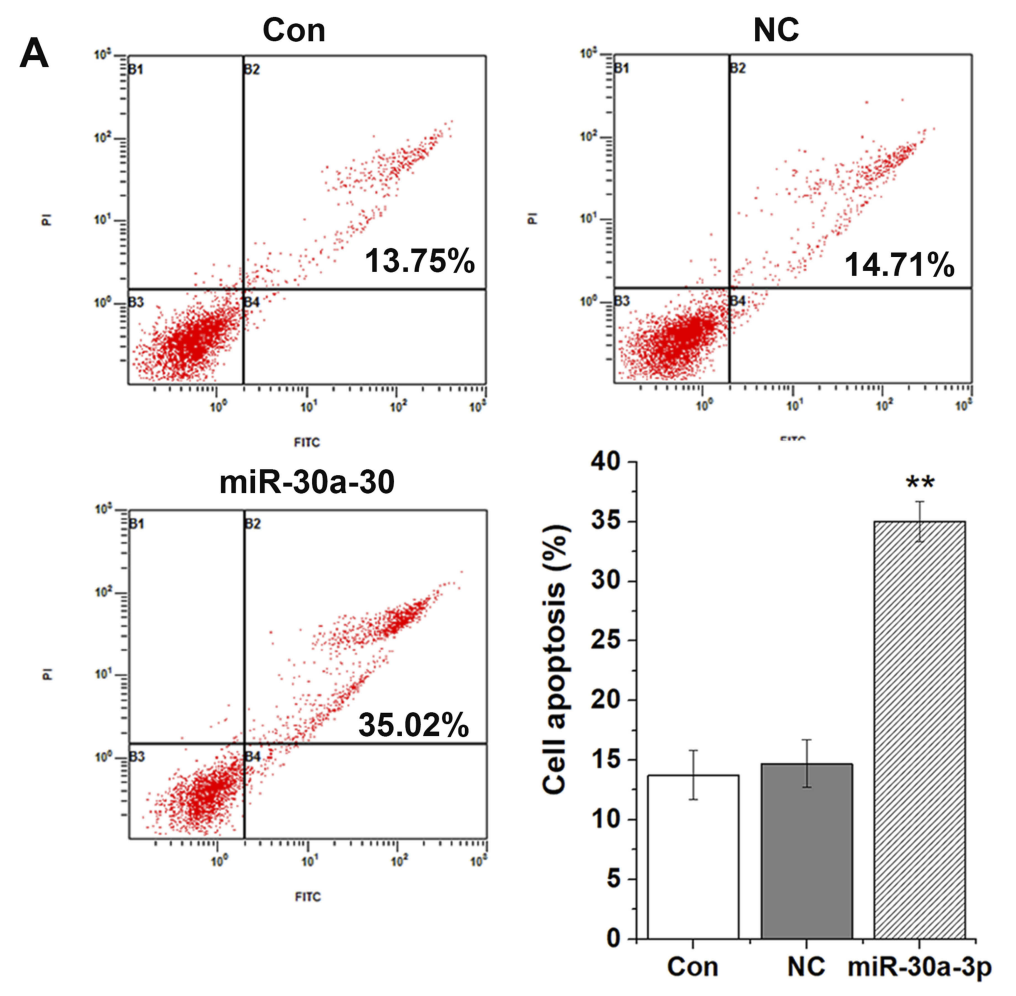

B
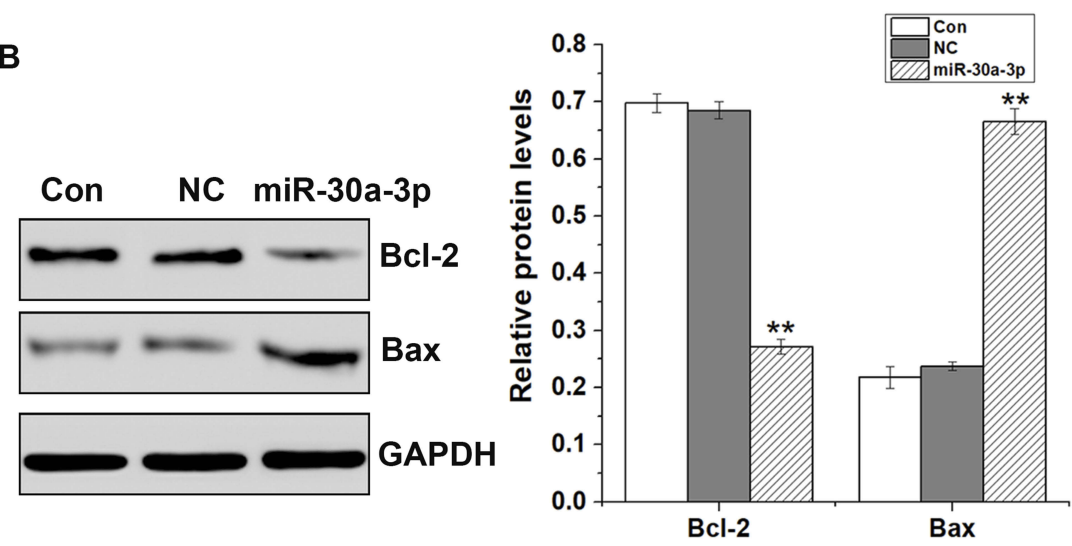

Figure 4 miR-30a-3p induces apoptosis in A549 cells. (A) Cell apoptosis was detected by Annexin VI/propidium iodide combined labeling flow cytometry in A549 cells at 48 hrs after transfection with miR-30a-3p. Apoptotic evaluation was carried out by the percentage of the apoptotic cell number of the total cell number. (B) Expression of cell apoptosis-associated proteins in A549 cells 48 hrs after transfection of miR-30a-3p by Western blot analysis. Data are reported as mean \pm SD ( $n=3$ ). $* * P<0.1$ versus NC group. Abbreviations: Con, control group; NC, cells transfected with miR-control vector group; miR-30a-3p, cells transfected miR-30a-3p vector group.

downregulated in hepatocellular carcinoma and acts as a tumor suppressor via regulation of cell proliferation and apoptosis. ${ }^{14}$ In breast cancer, miR-30a could suppress breast cancer cell migration and invasion and overexpression of miR-30a is associated with improved survival of breast cancer patients. $^{26,27}$ In line with most observations, our results demonstrated that overexpression of miR-30a-3p in A549 cells dramatically reduced the proliferation and induced the apoptosis of A549 cells.

It has been reported that DNMTs-mediated hypermethylation of promoters is one of the primary reason for inactivation of TSGs. Hypermethylation is responsible for the inactivation of TSGs involved in tumorigenesis. ${ }^{28}$ DNMT3a, which act as other DNMT family members, participated in tumorgenesis, metastasis and differentiation. ${ }^{29}$ In the present study, we found that miR-30a-3p was downregulated in lung cancer A549 cells, and overexpression of miR30a-3p inhibited the DNMT3a expressions in A549 cells. As previously mentioned, p38 MAPKs were found to up-regulated in the DNMT3a depletion cells, which suppresses tumor formation by negatively regulating cell proliferation and cycle progression, or by inducing apoptosis. ${ }^{30}$ Our 
A

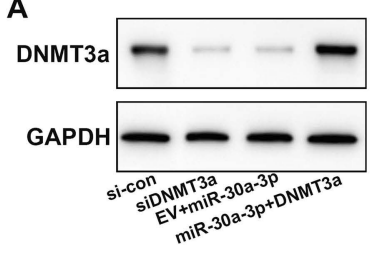

D

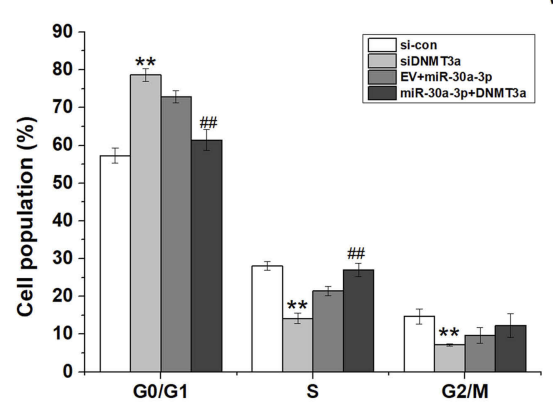

$\mathbf{F}$
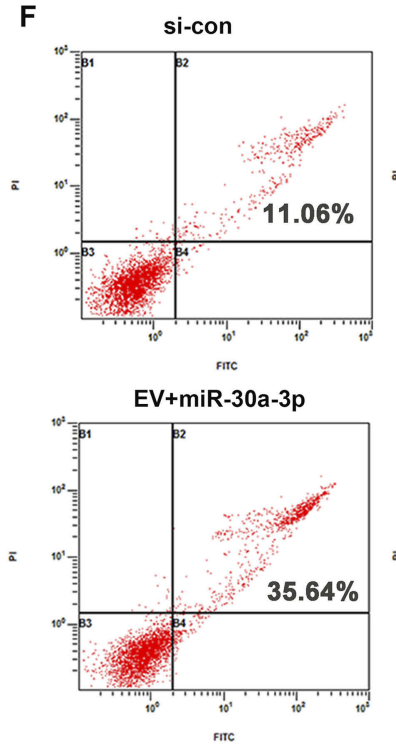
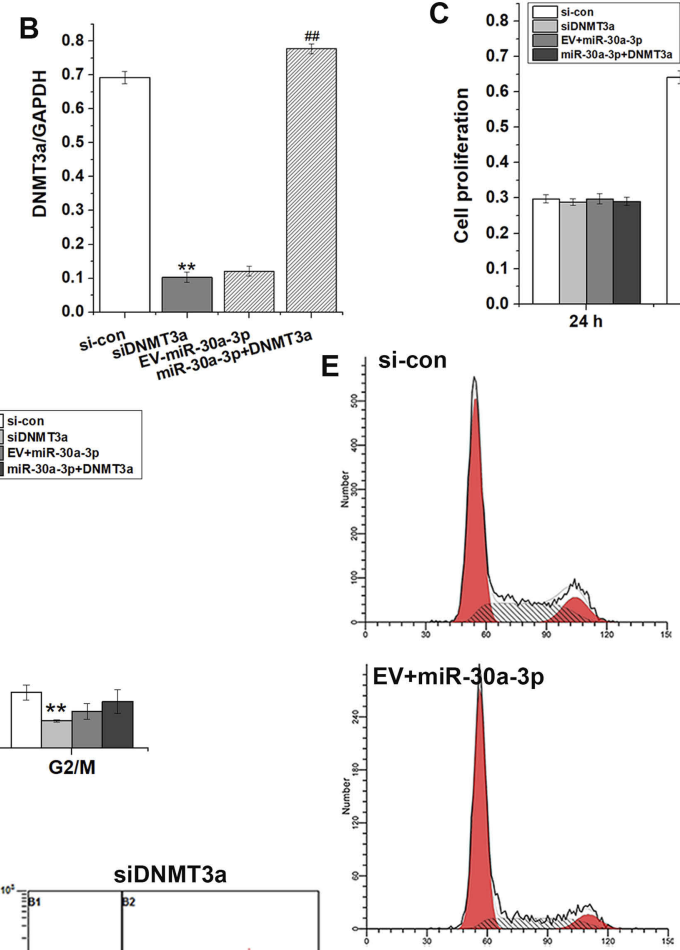

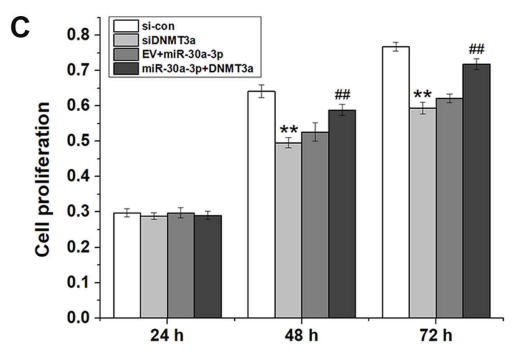

siDNMT3a
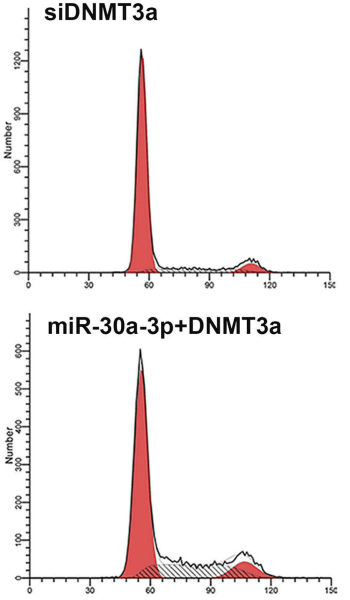

G
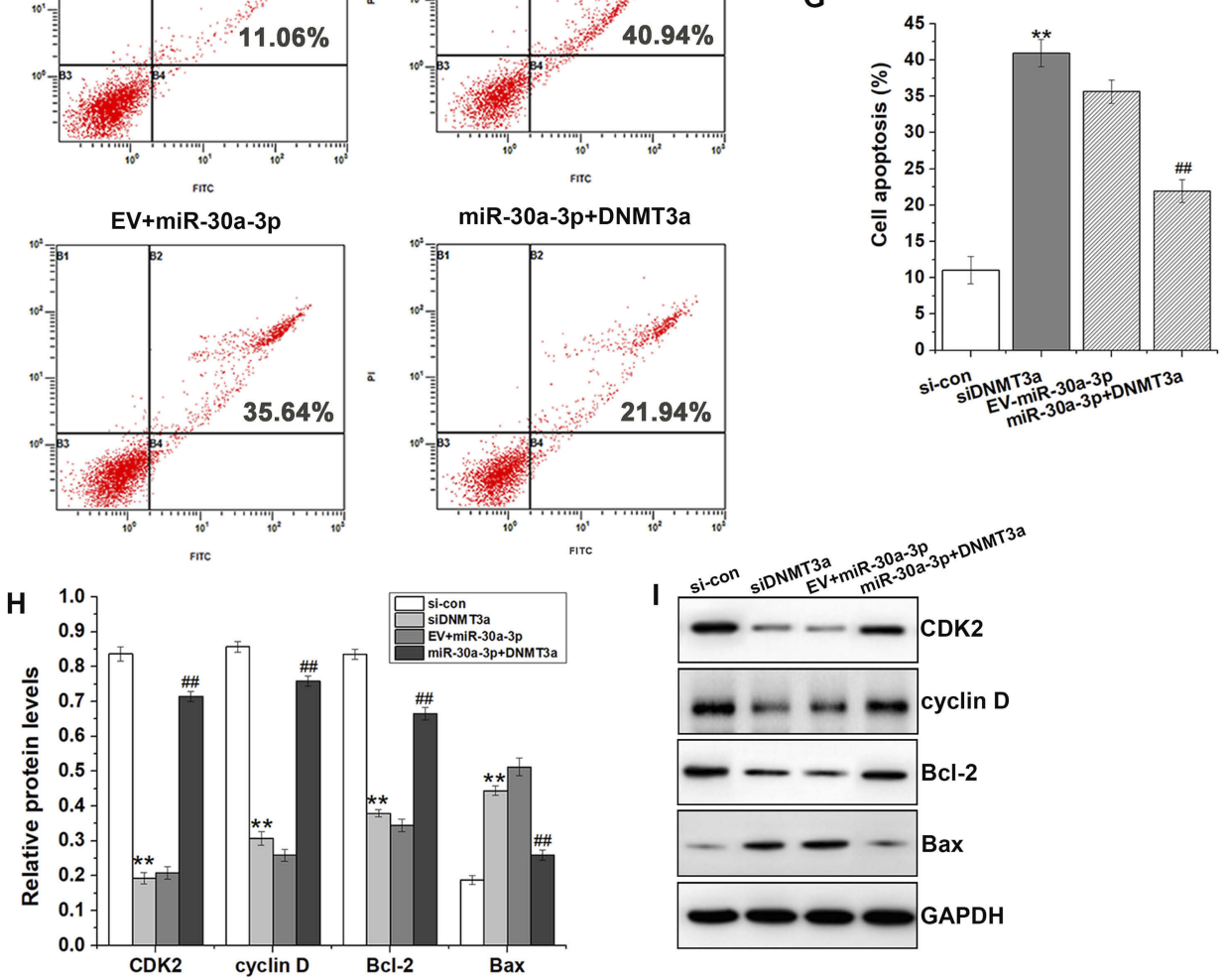

Figure 5 Silencing DNMT3a suppresses lung cancer cell proliferation and induces GI-phase arrest and cell apoptosis in A549 cells, and upregulating DNMT3a induces lung cancer cell proliferation and cycle progression and inhibits cell apoptosis in overexpression miR-30a-3p A549 cells. (A, B) Analysis of protein expression of DNMT3a in A549 cells at 48 hrs after transfection. (C) The effect of DNMT3a knockdown or overexpression on the proliferation of A549 cells were evaluated by MTT assay at 24,48 and 72 hrs. (D, E) The cell cycle was determined in A549 cells at 48 hrs after DNMT3a knockdown or overexpression. (F, G) Cell apoptosis was detected in A549 cells at $48 \mathrm{hrs}$ after DNMT3a knockdown or overexpression. (H, I) Analysis of protein expression associated with cell cycle and apoptosis in A549 cells at 48 hrs after transfection. Data are reported as mean $\pm S D(n=3)$. ${ }^{* * P}<00.1$ versus si-con group. ${ }^{\#} P<0.01$ versus $E V+$ miR-30a-3p group.

Abbreviations: Si-con, cells transfected with DNMT3a empty vector group; siDMNT3a, cells transfected with DNMT3a interference vector; EV+miR-30a-3p, cells transfected with miR-30a-3p overexpression vector and DNMT3a empty vector group; miR-30a-3p+DNMT3a, cells transfected miR-30a-3p and DNMT3a overexpression vector group. 
results showed that p-38 MAPK pathway was activated in A549 cells overexpression miR-30a-3p or siDNMT3a, resulting in inhibition of cell proliferation and cell cycle arrest. Evidence indicates that activation of p38-involved cell cycle regulates expression of p21CIP1, p27KIP1, cyclin $\mathrm{D}$ and CDKs. ${ }^{31,32} \mathrm{p} 21 \mathrm{CIP} 1$ and $\mathrm{p} 27 \mathrm{KIP} 1$ are typical tumor suppressors that regulate $\mathrm{G} 0$ to $\mathrm{S}$ phase transitions by regulating the activity of CDKs. ${ }^{33}$ Upregulation of $\mathrm{p} 27 \mathrm{KIP} 1$ and p21CIP1 are often induced by cell-cell contact-dependent fashion to inhibit cyclin-D and CDKs and hence promotes the inhibition of cell proliferation. ${ }^{34}$ In the present study, overexpression of miR-30a-3p or siDNMT3A inhibited DNMT3A expression, which leads to p38 activation. Next, we verified the effects of $\mathrm{p} 38$ on the downstream target genes p21CIP1, p27KIP1, cyclin D and CDK2, which are key transcriptional factors in the G0/S phase. From these results, we noted an increased expression of p21CIP1, p27KIP1 and a reduced expression of cyclin D and CDK in A549 cells transfected with miR-30a-3p.

Moreover, to explore the effect of DNMT3a and miR$30 a-3 p$ in the apoptosis of lung cancer A549 cells, we evaluated Bcl-2 and Bax protein expression levels, and suggested that miR-30a-3p inhibited the expression of DNMT3a which plays a critical role in the regulation of cell apoptosis. Bcl-2 is a proto-oncogene does not trigger cell proliferation but elevates the survival ability of cells under deleterious conditions. ${ }^{35}$ Bax as pro-apoptotic member can initiate cell death pathways, although it has similar sequence homology with Bcl-2. ${ }^{36}$ The ratio of Bcl-2 and Bax determines the response to a death signal via modulating membrane permeability transition pore opening. Our results indicate that miR-30a-3p can inhibit A549 cell apoptosis by upregulation of $\mathrm{Bcl}-2$ and downregulation of Bax.

In addition, in order to further validate our experiments, both miR-30a-3p and DNMT3a were transfected with A549 cells; the results suggested that overexpression of DNMT3a induced the growth of lung cancer cells and suppressed miR-30a-3p expression inhibited cell proliferation and included cell cycle arrest and apoptosis. The results support our findings that miR-30a-3p targets DNMT3a and suppresses lung cancer cell growth in the opposite direction.

In conclusion, we explored the roles of miR-30a-3p and its target gene DNMT3a in the proliferation, cell cycle and apoptosis of A549 cells. Those findings indicate that miR$30 \mathrm{a}-3 \mathrm{p}$ might act as a novel tumor suppressor via blocking the proliferation of lung cancer cells through the p38
MAPK pathway through targeting DNMT3a. Our findings highlight the functional association of miR-30a-3p, provide new insight into the regulatory network of the cell proliferation, cell cycle and apoptosis and open possibilities for future therapeutic interventions.

\section{Availability of data and materials}

All data generated or analyzed during the present study are included in this published article.

\section{Disclosure}

The authors report no conflicts of interest in this work.

\section{References}

1. Klose RJ, Bird AP. Genomic DNA methylation: the mark and its mediators. Trends Biochem Sci. 2006;31(2):89-97. doi:10.1016/j. tibs.2005.12.008

2. Li Y, Tollefsbol TO. Impact on DNA methylation in cancer prevention and therapy by bioactive dietary components. Curr Med Chem. 2010;17(20):2141-2151.

3. Goldberg AD, Allis CD, Bernstein E. Epigenetics: a landscape takes shape. Cell. 2007;128(4):635-638. doi:10.1016/j.cell.2007.02.006

4. Zhao Z, Wu Q, Cheng J, Qiu X, Zhang J, Fan H. Depletion of DNMT3A suppressed cell proliferation and restored PTEN in hepatocellular carcinoma cell. J Biomed Biotechnol. 2010;2010:737535. doi:10.1155/2010/737535

5. Shah MY, Licht JD. DNMT3A mutations in acute myeloid leukemia Nat Genet. 2011;43(4):289-290. doi:10.1038/ng0411-289

6. Wang Y, Xie Y, Li X, et al. MiR-876-5p acts as an inhibitor in hepatocellular carcinoma progression by targeting DNMT3A. Pathol Res Pract. 2018;214(7):1024-1030. doi:10.1016/j.prp.2018. 04.012

7. Zhao X, Deng R, Wang Y, et al. Twist1/Dnmt3a and miR186 establish a regulatory circuit that controls inflammation-associated prostate cancer progression. Oncogenesis. 2017;6(4):e315. doi:10.1038/ oncsis. 2017.16

8. Lin RK, Hsu HS, Chang JW, Chen CY, Chen JT, Wang YC. Alteration of DNA methyltransferases contributes to $5^{\prime} \mathrm{CpG}$ methylation and poor prognosis in lung cancer. Lung Cancer. 2007;55 (2):205-213. doi:10.1016/j.lungcan.2006.10.022

9. Kim BG, Gao MQ, Kang S, et al. Mechanical compression induces VEGFA overexpression in breast cancer via DNMT3A-dependent miR-9 downregulation. Cell Death Dis. 2017;8(3):e2646. doi:10. 1038/cddis.2017.73

10. Bartel DP. MicroRNAs: genomics, biogenesis, mechanism, and function. Cell. 2004;116(2):281-297. doi:10.1016/s0092-8674(04) 00045-5

11. Wiemer EA. The role of microRNAs in cancer: no small matter. Eur J cancer. 2007;43(10):1529-1544. doi:10.1016/j.ejca.2007.04.002

12. Shen Q, Bae HJ, Eun JW, et al. MiR-101 functions as a tumor suppressor by directly targeting nemo-like kinase in liver cancer. Cancer Lett. 2014;344(2):204-211. doi:10.1016/j.canlet.2013.10.030

13. Pang JC, Kwok WK, Chen Z, Ng HK. Oncogenic role of microRNAs in brain tumors. Acta Neuropathol. 2009;117(6):599-611. doi:10.1007/s00401-009-0525-0

14. Wang W, Lin H, Zhou L, et al. MicroRNA-30a-3p inhibits tumor proliferation, invasiveness and metastasis and is downregulated in hepatocellular carcinoma. Eur J Surg Oncol. 2014;40(11):15861594. doi:10.1016/j.ejso.2013.11.008 
15. Mathew LK, Lee SS, Skuli N, et al. Restricted expression of miR30c-2-3p and miR-30a-3p in clear cell renal cell carcinomas enhances HIF2alpha activity. Cancer Discov. 2014;4(1):53-60. doi:10.1158/ 2159-8290.CD-13-0291

16. Zhang HD, Jiang LH, Sun DW, Li J, Tang JH. miR-30a inhibits the biological function of breast cancer cells by targeting Notch1. Int J Mol Med. 2017;40(4):1235-1242. doi:10.3892/ijmm.2017.3084

17. Liu X, Ji Q, Zhang C, et al. miR-30a acts as a tumor suppressor by double-targeting COX-2 and BCL9 in H. pylori gastric cancer models. Sci Rep. 2017;7(1):7113. doi:10.1038/s41598-017-07193-w

18. Lee H, Park CS, Deftereos G, et al. MicroRNA expression in ovarian carcinoma and its correlation with clinicopathological features. World J Surg Oncol. 2012;10:174. doi:10.1186/1477-7819-10-198

19. Qi B, Wang Y, Chen ZJ, et al. Down-regulation of miR-30a-3p/5p promotes esophageal squamous cell carcinoma cell proliferation by activating the Wnt signaling pathway. World J Gastroenterol. 2017;23(45):7965-7977. doi:10.3748/wjg.v23.i45.7965

20. Yanaihara N, Caplen N, Bowman E, et al. Unique microRNA molecular profiles in lung cancer diagnosis and prognosis. Cancer Cell. 2006;9(3):189-198. doi:10.1016/j.ccr.2006.01.025

21. Fan MJ, Zhong YH, Shen W, et al. MiR-30 suppresses lung cancer cell 95D epithelial mesenchymal transition and invasion through targeted regulating Snail. Eur Rev Med Pharmacol Sci. 2017;21 (11):2642-2649.

22. Ling XH, Chen ZY, Luo HW, et al. BCL9, a coactivator for Wnt/ beta-catenin transcription, is targeted by miR-30c and is associated with prostate cancer progression. Oncol Lett. 2016;11(3):2001-2008. doi:10.3892/ol.2016.4161

23. Ma Y, Zhang P, Yang J, Liu Z, Yang Z, Qin H. Candidate microRNA biomarkers in human colorectal cancer: systematic review profiling studies and experimental validation. Int J Cancer. 2012;130(9):20772087. doi:10.1002/ijc. 26232

24. Yan LX, Huang XF, Shao Q, et al. MicroRNA miR-21 overexpression in human breast cancer is associated with advanced clinical stage, lymph node metastasis and patient poor prognosis. RNA. 2008;14(11):2348-2360. doi:10.1261/rna.1034808

25. Kano M, Seki N, Kikkawa N, et al. miR-145, miR-133a and miR133b: tumor-suppressive miRNAs target FSCN1 in esophageal squamous cell carcinoma. Int $J$ Cancer. 2010;127(12):2804-2814. doi:10.1002/ijc. 25284
26. Kawaguchi T, Yan L, Qi Q, et al. Overexpression of suppressive microRNAs, miR-30a and miR-200c are associated with improved survival of breast cancer patients. Sci Rep. 2017;7(1):15945. doi:10.1038/s41598-017-16112-y

27. Cheng CW, Wang HW, Chang CW, et al. MicroRNA-30a inhibits cell migration and invasion by downregulating vimentin expression and is a potential prognostic marker in breast cancer. Breast Cancer Res Treat. 2012;134(3):1081-1093. doi:10.1007/s10549-012-2034-4

28. Wang D, Cui W, Wu X, et al. RUNX3 site-specific hypermethylation predicts papillary thyroid cancer recurrence. Am J Cancer Res. 2014;4(6):725-737.

29. Ma QL, Wang JH, Wang YG, et al. High IDH1 expression is associated with a poor prognosis in cytogenetically normal acute myeloid leukemia. Int J Cancer. 2015;137(5):1058-1065. doi:10.1002/ijc. 29395

30. Dolado I, Swat A, Ajenjo N, De Vita G, Cuadrado A, Nebreda AR. p38alpha MAP kinase as a sensor of reactive oxygen species in tumorigenesis. Cancer Cell. 2007;11(2):191-205. doi:10.1016/j.ccr. 2006.12.013

31. Saha K, Adhikary G, Kanade SR, Rorke EA, Eckert RL. p38delta regulates p53 to control p21Cip1 expression in human epidermal keratinocytes. J Biol Chem. 2014;289(16):11443-11453. doi:10.10 74/jbc.M113.543165

32. Wang F, Wang Q, Zhou ZW, et al. Plumbagin induces cell cycle arrest and autophagy and suppresses epithelial to mesenchymal transition involving PI3K/Akt/mTOR-mediated pathway in human pancreatic cancer cells. Drug Des Devel Ther. 2015;9:537-560. doi:10.2147/DDDT.S73689

33. Chu IM, Hengst L, Slingerland JM. The Cdk inhibitor p27 in human cancer: prognostic potential and relevance to anticancer therapy. Nature Rev Cancer. 2008;8(4):253-267. doi:10.1038/nrc2347

34. Noseda M, Chang L, McLean G, et al. Notch activation induces endothelial cell cycle arrest and participates in contact inhibition: role of p21Cip1 repression. Mol Cell Biol. 2004;24(20):8813-8822. doi:10.1128/MCB.24.20.8813-8822.2004

35. Vaux DL, Cory S, Adams JM. Bcl-2 gene promotes haemopoietic cell survival and cooperates with c-myc to immortalize pre-B cells. Nature. 1988;335(6189):440-442. doi:10.1038/335440a0

36. Suzuki M, Youle RJ, Tjandra N. Structure of bax: coregulation of dimer formation and intracellular localization. Cell. 2000;103 (4):645-654. doi:10.1016/s0092-8674(00)00167-7

\section{Publish your work in this journal}

OncoTargets and Therapy is an international, peer-reviewed, open access journal focusing on the pathological basis of all cancers, potential targets for therapy and treatment protocols employed to improve the management of cancer patients. The journal also focuses on the impact of management programs and new therapeutic agents and protocols on patient perspectives such as quality of life, adherence and satisfaction. The manuscript management system is completely online and includes a very quick and fair peer-review system, which is all easy to use. Visit http://www.dovepress.com/ testimonials.php to read real quotes from published authors. 